The Impact of Proposed Changes to ICD-11 on Estimates of PTSD Prevalence and Comorbidity

Blair E. Wisco ${ }^{\mathrm{a}}$, Mark W. Miller ${ }^{\mathrm{b}}$, Erika J. Wolf ${ }^{\mathrm{b}}$, Dean Kilpatrick ${ }^{\mathrm{c}}$, Heidi S. Resnick ${ }^{\mathrm{c}}$, Christal L. Badour $^{\mathrm{c}, 1}$, Brian P. Marx ${ }^{\mathrm{b}}$, Terence M. Keane ${ }^{\mathrm{b}}$, Raymond C. Rosen ${ }^{\mathrm{d}}$, \& Matthew J. Friedman ${ }^{\mathrm{e}}$

a. Department of Psychology, University of North Carolina at Greensboro, Postal Address:

UNCG Psychology, PO Box 26170, Greensboro, North Carolina, 27402 USA, email: bewisco@uncg.edu

b. VA National Center for PTSD, Boston, MA, and Department of Psychiatry, Boston University School of Medicine, Postal Address: National Center for PTSD (116B-2), VA Boston Healthcare System, 150 South Huntington Avenue, Boston, Massachussetts, 02130, USA, emails: Mark.Miller5@va.gov, Erika.Wolf@va.gov, Brian.Marx@va.gov,

\title{
Terry.Keane@va.gov
}

c. Department of Psychiatry and Behavioral Sciences, Medical University of South Carolina at Charleston, Postal Address: 67 President Stress, MSC 861, Charleston, South Carolina, 29425, USA, emails: kilpatdg@musc.edu, resnickh@musc.edu, christal.badour@uky.edu d. New England Research Institutes, Postal Address: 480 Pleasant Street, Watertown, Massachusetts, 02472, USA, email: RRosen@neriscience.com

e. VA National Center for PTSD, White River Junction, Vermont, \& Departments of Psychiatry and Pharmacology and Toxicology, Geisel School of Medicine at Dartmouth, 
Postal Address: National Center for PTSD, VA Medical Center (116D), 215 North Main Street, White River Junction, Vermont, 05009 , USA, email: matthew.friedman@va.gov

1. Dr. Christal Badour is now at the Department of Psychology, University of Kentucky, Postal Address: Department of Psychology, 106-B Kastle Hall, Unviersity of Kentucky, Lexington, KY, 40506, USA, email: christal.badour@uky.edu

* Correspondence concerning this article should be addressed to Mark W. Miller, National Center for PTSD (116B-2), VA Boston Healthcare System, 150 South Huntington Avenue, Boston, MA 02130. E-mail: mark.miller5@va.gov. 


\begin{abstract}
The World Health Organization's posttraumatic stress disorder (PTSD) work group has published a proposal for the forthcoming edition of the International Classification of Diseases $(I C D-11)$ that would yield a very different diagnosis relative to $D S M-5$. This study examined the impact of the proposed $I C D-11$ changes on PTSD prevalence relative to the $I C D-10$ and $D S M-5$ definitions and also evaluated the extent to which these changes would accomplish the stated aim of reducing the comorbidity associated with PTSD. Diagnostic prevalence estimates were compared using a U.S. national community sample and two U.S. Department of Veterans Affairs clinical samples. The $I C D$ - 11 definition yielded prevalence estimates 10 to 30 percent lower than DSM-5 and 25 and 50 percent lower than $I C D-10$ with no reduction in the prevalence of common comorbidities. Findings suggest that by constraining the diagnosis to a narrower set of symptoms, the proposed $I C D-11$ criteria set would substantially reduce the number of individuals with the disorder. These findings raise doubt about the extent to which the ICD-11 proposal would achieve the aim of reducing comorbidity associated with PTSD and highlight the public health and policy implications of such a redefinition.
\end{abstract}

Keywords: PTSD; trauma; DSM-5; ICD-11; diagnosis; diagnostic criteria 


\section{The Impact of Proposed Changes to ICD-11 on Estimates of PTSD Prevalence and}

\section{Comorbidity}

\section{Introduction}

Mental disorders are defined and classified according to two systems: the Diagnostic and Statistical Manual of Mental Disorders, published by the American Psychiatric Association and now in its fifth edition (DSM-5; APA, 2013), and the World Health Organization's International Classification of Diseases, now in its tenth edition (ICD-10; WHO, 1992). At first glance, the two systems look quite similar-they comprise an almost identical collection of major diagnoses, they classify them under similar categories, and the codes for individual diagnoses are used interchangeably in medical record and billing systems throughout the world. Upon closer inspection, however, important distinctions become evident for certain disorders, with posttraumatic stress disorder (PTSD) showing some of the most striking differences between the two systems. Though both define PTSD as a constellation of symptoms including reexperiencing, avoidance, and hyperarousal, among others, that emerge following exposure to trauma, they differ with respect to the definition of traumatic events, the requisite number, combination, and duration of symptoms, and whether functional impairment is required. It is perhaps not surprising then that studies that have compared PTSD diagnostic prevalence estimates using the two definitions have yielded higher levels of discordance for this diagnosis relative to others. For example, Andrews et al. (1999) compared prevalence estimates for common mental disorders using $I C D-10$ versus $D S M-I V$ criteria and found that PTSD showed the highest level of disagreement of all the anxiety disorders. In that large epidemiological survey sample, the DSM-IV criteria yielded a 3.0\% 12-month prevalence estimate for PTSD whereas the $I C D-10$ criteria resulted in a $6.9 \%$ estimate. Subsequent analyses revealed the 
primary source of the discrepancy to be attributable to the DSM-IV requirement that the symptoms cause clinically significant distress or impairment (Peters et al., 1999).

Though DSM-IV and ICD-10 have co-existed for 20 years, few other studies comparing diagnostic prevalence estimates can be found in the scientific literature. Such comparisons are important given policy changes that may dramatically increase the use of the ICD in the United States. The U.S. Health Insurance Portability and Accountability Act (HIPPA) of 1996, best known for setting new standards for patient privacy in the U.S., also established that ICD codes would be required for all billing and reimbursement transactions covered by the law. Though implementation has been slow, as of October 2015 the U.S. Centers for Medicare and Medicaid Services require $I C D$ - 10 coding for all services. Further, the U.S. government, as a participating member of the WHO, is obligated to implement $I C D-11$ when it is finalized, and as Reed (2010, p. 458) noted, "it would be extremely difficult to justify the U.S. continuing not to use the same system that has been adopted as the standard by the rest of the world." The development and existence of two distinct PTSD diagnoses has the potential to complicate use and interpretation of the PTSD diagnosis among clinicians, researchers, and policy-makers alike.

The WHO Working Group on the "Classification of Disorders Specifically Associated with Stress" has published several papers outlining their proposal for revisions to the PTSD diagnosis in ICD-11 (e.g., Maercker, Brewin, Bryant, Cloitre, Reed, et al., 2013; Maercker, Brewin, Bryant, Cloitre, van Ommeren, et al., 2013). Although some parts of the proposal outlined in these papers paralleled changes made to PTSD for DSM-5 (e.g., moving the diagnosis out of the anxiety disorders and into its own class of stress-related conditions) and the working definition of trauma for $I C D-11$ remains a close approximation to the $D S M-5$ Criterion A, other modifications would represent a more radical departure from DSM-5. Specifically, Maercker, 
Brewin, Bryant, Cloitre, Reed, et al.’s (2013) and Maercker, Brewin, Bryant, Cloitre, van Ommeren, et al.'s (2013) proposal (outlined also by Brewin, 2013) seeks to reduce the large number of "non-specific symptoms" of PTSD that overlap with symptoms of other disorders, such as mood and anxiety disorders, thereby increasing the discriminant validity of the diagnosis. Following similar recommendations made previously (Brewin et al., 2009; Rosen et al., 2010), the new proposal would narrow the scope of the construct by focusing on three core elements: reexperiencing of the trauma, avoidance of reminders of the event, and a heightened perception of threat and arousal. Maercker Brewin, Bryant, Cloitre, van Ommeren, et al. (2013) noted that the objectives of these changes would be to reduce the high rate of comorbidity with other diagnoses, reduce the number of symptom combinations that are mathematically possible under the DSM-5 definition, and enhance the "clinical utility" of the diagnosis, which Brewin (2013, p. 557) noted "specifically refers to ease of use in nonspecialist, minimally resourced, and nonEnglish-speaking settings."

In contrast to DSM-5, which provides a list of symptoms and specifies a minimum number of requisite symptoms for each diagnostic criterion, $I C D-11$ would provide a narrative description of each of the three core elements. Specifically, re-experiencing symptoms would be defined as "reexperiencing the traumatic event(s) in the present in the form of vivid intrusive memories accompanied by fear or horror, flashbacks, or nightmares," avoidance defined as "avoidance of thoughts and memories of the event(s) or avoidance of activities or situations reminiscent of the event(s)," and heightened perception of threat and arousal defined as "a state of perceived current threat in the form of excessive hypervigilance or enhanced startle reactions" (Maercker, Brewin, Bryant, Cloitre, van Ommeren, et al., 2013). The members of the ICD-11 work group have operationalized this definition as endorsement of at least one re-experiencing 
symptom (i.e., flashbacks or nightmares), one avoidance symptom (i.e., avoidance of internal or external reminders) and one hyperarousal symptom (i.e., hypervigilance or exaggerated startle; Cloitre et al., 2013). The proposed $I C D-11$ criteria also include a new functional impairment requirement which, as noted earlier, was absent from the $I C D$ - 10 diagnosis.

The $I C D-11$ proposal would therefore omit all seven of the $D S M-5$ "negative alterations in cognitions and mood" symptoms and substantially narrow the definitions of re-experiencing and hyperarousal symptoms. Specifically, the $I C D-11$ definition provides a stricter definition of intrusive memories, limited to "vivid intrusive memories accompanied by fear or horror" (DSM5 B1), omits two other re-experiencing symptoms (emotional or physiological reactivity to trauma reminders; DSM-5 B4 and B5), and omits four "nonspecific" hyperarousal symptoms (irritability, reckless or self-destructive behavior, concentration difficulties, and sleep disturbance; $D S M-5 \mathrm{E} 1, \mathrm{E} 2, \mathrm{E} 5$, and E6). The primary changes relative to $I C D-10$ would be the more narrow definition of intrusive thoughts, elimination of emotional distress or physiological reactivity when reminded of the traumatic event, the removal of psychogenic amnesia, the omission of three non-specific symptoms (sleep disturbance, anger, and concentration difficulties), and again, the addition of the functional impairment requirement.

To summarize, the proposed ICD-11 PTSD definition stands in contrast with the broader DSM-5 conceptualization and the two approaches represent very different views of the disorder and how to achieve a clinically useful diagnosis. Prior studies that have compared diagnostic prevalence estimates using the two approaches have yielded mixed results. Stein and colleagues (Stein et al., 2014) examined prevalence estimates derived from using Composite International Diagnostic Interview (CIDI) in the multi-national population-based World Mental Health Surveys, and found equivalent PTSD prevalence estimates using the DSM-5 (3.0\%) and ICD-11 
(3.2\%) algorithms. However, this study was based on a DSM-IV referenced assessment that did not reflect the new symptoms or other important changes evident in DSM-5 and was based on retrospective reports of lifetime symptoms. In contrast, O’Donnell and colleagues (2014) used a modified version of the Clinician Administered PTSD Scale (Blake et al., 1995) that incorporated the new symptoms into the interview to estimate current PTSD prevalence in a random sample of hospital patients 72 months postdischarge and found that significantly fewer individuals would meet criteria under ICD-11 compared with DSM-5 (3.3\% versus $6.7 \%$, respectively). They also compared the proportions of cases with PTSD who met criteria for comorbid major depression and found that the more restrictive $I C D-11$ definition did not significantly reduce depression comorbidity. These results are broadly consistent with prior findings indicating that eliminating overlapping symptoms from the DSM-IV PTSD definition does not reduce depression comorbidity (Elhai et al., 2008; Grubaugh et al., 2010). Other studies have examined comorbidity among individuals whose PTSD diagnostic status was discordant (i.e., they met criteria for PTSD according to $D S M-I V$ but not $I C D-11$ or vice versa). Individuals who met $I C D-11$ criteria only were significantly less likely to be depressed than the $D S M-I V$ only cases (Morina et al., 2014; Stammel et al., 2015). However, these statistical comparisons excluded those who meet criteria for PTSD according to both diagnostic systems, which is the majority of individuals with PTSD according to $I C D-11$. Another relevant comparison is with the proportion of individuals who meet criteria for PTSD according to $I C D-11$ (whether or not they also meet criteria according to $D S M-I V$ ) and those who only meet criteria according to $D S M-I V$ (the group who would lose the diagnosis under ICD-11). Both Morina and colleagues (2014) and Stammel and colleagues (2015) examined this question and found that the ICD-11 group had higher or comparable rates of depression compared with the DSM-IV only group 
(49.7\% vs. 43.8\%; Morina et al., 2014; Sample 1: 79.3\% vs. 79.0\%,Sample 2: 89.1\% vs. 84.2\%;

Stammel et al., 2015), again, implying that the $I C D-11$ revision may not meet the aim of lowering psychiatric comorbidity by removing non-specific PTSD symptoms from the criteria set.

Given the wide-reaching implications of a revision that could substantially alter diagnostic prevalence estimates, we compared $D S M-5$ with $I C D-10$ and $I C D-11$ PTSD estimates in a U.S. national community sample and a U.S. Department of Veterans Affairs clinical sample. Then, in a third sample that was more comprehensively assessed for an array of other disorders that commonly co-occur with PTSD, we examined the extent to which proposed changes to ICD11 would reduce such comorbidity (Maercker, Brewin, Bryant, Cloitre, Reed, et al., 2013;

Maercker, Brewin, Bryant, Cloitre, van Ommeren, et al., 2013).

\section{Methods}

\subsection{Participants}

\subsubsection{Sample 1-On-line Survey of a U.S. National Community Sample. Table 1}

provides demographic characteristics of each of the three samples included in this paper. Sample 1 participants were recruited from an online probability-based panel representative of the U.S. adult population maintained by an internet survey research firm. This sample and study methods were described at length in prior publications based on this dataset (Kilpatrick et al., 2013; Miller, Wolf, Kilpatrick, et al., 2013) and so will be summarized briefly here. Eligible panel participants were stratified on the basis of sex and age categories within the U.S. Census breakdown of the population. Though this method does not yield a true national probability sample (since an estimated $20 \%$ of households do not have internet access) it does provide a diverse sample that is generally demographically and geographically representative of U.S. 
adults. 3,756 individuals connected to the survey website and $92 \%(n=3,457)$ of those agreed to participate. 2,953 completed the survey representing $85.4 \%$ of those who agreed to participate and a $78.6 \%$ of those who accessed the URL. Data are not available regarding how many individuals received invitations to participate or the proportion of those receiving invitations that accessed the website (Kilpatrick et al., 2013).

As reported previously (Kilpatrick et al., 2013; Wolf et al., 2015), participants reported exposure to a wide range of $D S M-5$ traumatic events including being a victim of physical or sexual assault (53.1\%), death of a family member or close friend due to an accident, violence, or disaster (51.8\%), natural disaster (50.5\%), accident/fire $(48.3 \%)$, witnessing a physical or sexual assault $(33.2 \%)$, threat or injury to a family member or close friend due to violence/accident/disaster (32.4\%), and witnessing a dead body unexpectedly (22.6\%). Combat or war zone exposure was endorsed by $7.8 \%$. The modal number of DSM-5 Criterion A events within the full sample was three, with a mean of 3.3 and standard deviation of 2.3. Analyses were based on 2,695 participants who completed the simple and complex PTSD sections of the survey. All survey data from Sample 1 were weighted by age, sex, and race/ethnicity based on 2010 U.S. Census data.

2.1.2. Sample 2-On-line Survey of U.S. Military Veterans. Sample 2 was comprised of U.S. military veterans who were recruited from one of two recruitment sources. The first was a letter mailed to 700 veterans who had previously consented to be contacted for research studies. One hundred seven letters were returned for bad addresses. Of the remaining 593 potential participants, $123(21 \%)$ completed the survey. The second recruitment source was an emailed invitation to 278 veterans of Operations Enduring Freedom and Iraqi Freedom (OEF/OIF) who were enrolled in a PTSD registry, the Veterans' Afterdischarge Longitudinal Registry (Project 
VALOR; Rosen et al., 2012). Project VALOR was designed as a longitudinal patient registry of OEF/OIF veterans who have undergone a mental health evaluation in the Veterans Affairs healthcare system, with an overrepresentation of veterans with a PTSD diagnosis $(75 \%$ of the final sample) and of female veterans (50\% of final sample). Of the 278 invited veterans, 222 veterans $(80 \%)$ endorsed trauma exposure and completed the survey, yielding a total of 345 study participants from the two recruitment sources. After providing informed consent on-line, participants were then linked to the webpage with the survey questions. Twenty-two did not complete the symptom assessment and were excluded, yielding a final sample of 323 veterans ( $83 \%$ with a history of combat exposure). Of these, $75 \%$ had served during the OEF/OIF era, $15 \%$ in the Vietnam War era, $4 \%$ in the Operation Desert Storm era, and 1\% served in the Korean War or World War II eras.

2.1.3. Sample 3-Clinical Interview Sample of Veterans and Partners. Sample 3 was based on a cohort of 852 participants enrolled into one of two research protocols at U.S. Department of Veterans Affairs medical centers. This sample and the relevant recruitment and data collection methods have been described at length previously (e.g., Logue et al., 2013; Miller, Wolf, Logue, et al., 2013). Briefly, the first protocol enrolled trauma-exposed veterans who screened positive for DSM-IV PTSD during a telephone interview; the second recruited military veterans with trauma histories and their cohabitating partners. Four hundred and sixtynine veterans and 279 partners completed the structured diagnostic interviews, yielding a final sample size of 748 for these analyses. They reported exposure to a wide variety of traumatic events on the Traumatic Life Events Questionnaire (Kubany et al., 2000) and exposure to multiple events over the course of the lifespan was the norm. Events most frequently endorsed by male participants were combat (54.9\%), sudden and unexpected death of a loved one $(6.1 \%)$, 
and assault by acquaintance/stranger (5.4\%). For women, the most frequently endorsed events were childhood and/or adult sexual assault (19.3\%), childhood and/or adult physical abuse (15.0\%), and sudden and unexpected death of a loved one (14.4\%).

\subsection{Measures}

\subsubsection{Samples 1 and 2: National Stressful Events Survey (NSES; Kilpatrick et al.,}

2011). Participants in Samples 1 and 2 were administered the NSES to assess exposure to traumatic events and the 20 DSM-5-defined PTSD symptoms. Twenty-five close-ended questions assessed exposure to a range of events that would meet Criterion A 1 under the DSM-IV definition and/or Criterion A under the DSM-5 definition. Each symptom was assessed using a series of items that began by asking if the participant had "ever" experienced the symptom. Those who endorsed this question then indicated when they had last experienced it using an interval ranging from "during the past month" to "more than one year ago." Participants who endorsed a symptom in the past month were then asked to rate how bothered they had been by it using a Likert-like scale that ranged from 1 (not at all) to 5 (extremely). Following methods established for the PTSD Checklist (Weathers et al., 1993) which uses the same response options, only symptoms endorsed at a level of 3 ("moderately") or greater were coded as present for estimating diagnosis. For symptoms not explicitly linked to trauma (e.g., the majority of the DSM-5 criterion D and E symptoms), participants were also asked whether the symptom "began or got worse" after trauma and this item had to be answered affirmatively for the symptom to count towards a diagnosis. The symptom assessment was then followed by a series of questions assessing psychological distress and functional impairment. Coefficient alpha for the past-month symptoms in Sample 1 was 0.90 for the items corresponding to DSM-IV, 0.91 for DSM-5, 0.87 
for $I C D-10$, and 0.77 for $I C D-11$. Corresponding alphas for Sample 2 were 0.93 for $D S M-I V$, 0.94 for $D S M-5,0.92$ for $I C D-10$, and 0.87 for $I C D-11$.

2.2.2. Sample 3: Clinician Administered PTSD Scale (CAPS; Blake et al., 1995) and Structured Clinical Interview for DSM-IV (SCID-IV; First et al., 1994). In Sample 3, PTSD was assessed using the Clinician Administered PTSD Scale for DSM-IV, a 30-item structured diagnostic interview used to measure each of the 17 DSM-IV PTSD symptoms and functional impairment with each symptom rated on separate frequency and intensity scales. Other Axis I disorders were assessed using the Structured Clinical Interview for DSM-IV. All interviews were administered by experienced clinicians who received extensive training prior to data collection. Each interview was video-recorded and approximately $25 \%$ were later viewed by independent raters for purposes of maintaining quality control and evaluating inter-rater reliability. To minimize rater-drift and enhance interview quality, rating discrepancies were discussed in weekly team meetings throughout the course of data collection. Reliability statistics (kappas for past month diagnosis) for the diagnoses examined in these analyses were as follows: PTSD (0.74), alcohol abuse (0.72), alcohol dependence (0.56), generalized anxiety disorder (0.83), major depressive episode (0.86), panic disorder with agoraphobia (0.91), and panic disorder with or without agoraphobia (0.83).

\subsection{Operational definitions of PTSD}

2.3.1. ICD-11. As noted earlier, the working definition of trauma (e.g., Criterion A) for $I C D-11$ is highly similar to the DSM-5 definition so, to keep this factor constant across analyses, we applied the $D S M-5$ Criterion A definition in coding the $I C D-11$ diagnosis. Following the operationalization proposed by Cloitre et al. (2013), an ICD-11 diagnosis was coded positive when there was (a) exposure to a DSM-5 qualifying event accompanied by (b) at least one re- 
experiencing symptom (nightmares or flashbacks), (c) one avoidance symptom (avoidance of either internal or external trauma reminders), (d) one "sense of threat" symptom (hypervigilence or startle) and (e) significant distress or functional impairment as indexed by endorsement of at least one of four items assessing this criterion.

2.3.2. ICD-10. To facilitate cross-definition comparison we held the traumatic stressor definition constant in applying the $I C D-10$ algorithm. Thus, the $I C D-10$ diagnosis was coded positive when there was (a) exposure to a DSM-5 qualifying event, (b) at least one of four possible re-experiencing symptoms, (c) one avoidance symptom, and (d) either psychogenic amnesia or two or more hyperarousal symptoms endorsed at a level of 3 or greater. There was no separate distress or functional impairment requirement.

2.3.3. DSM-5. The DSM-5 diagnosis was coded positive when there was (a) exposure to a qualifying event, (b) at least one intrusion symptom, (c) one avoidance symptom, (d) two negative alterations of cognitions and mood symptoms, (e) two alterations in arousal and reactivity symptoms, and (f) significant distress or functional impairment as indexed by endorsement of at least one of four items assessing this criterion.

2.3.4. DSM-IV. In Sample 3, which used the CAPS, a DSM-IV diagnosis was coded positive when there was (a) exposure to a $D S M-I V$ qualifying event, (b) at least one intrusion symptom, (c) at least three numbing symptoms, (d) at least two hyperarousal symptoms scored at a frequency of one or greater (i.e., occurred at least once or twice in the past month) and an intensity of two or more (i.e., caused at least moderate distress) along with functional impairment.

\subsection{Statistical analyses}


First, we computed the prevalence of PTSD using the ICD-11,ICD-10, and DSM-5 definitions in Samples 1 and 2 and the $I C D-11, I C D-10$, and DSM-IV definitions in Sample 3. We then examined patterns of agreement and disagreement between ICD-11 and DSM-5, the two systems which will be used concurrently, in Samples 1 and 2 with McNemar's test, which is appropriate for tests of differences in a dichotomous variable between two related groups. Because clinical interview data were only available for Sample 3, comorbidity analyses were conducted in Sample 3 only. In Sample 3, we computed the prevalence of the four most common comorbidities relative to ICD-11 and DSM-IV PTSD diagnoses. We calculated these comorbidities for five different groups: all individuals who met DSM-IV PTSD criteria ("All $D S M-I V ")$, all individuals who met ICD-11 PTSD criteria ("All ICD-11"), individuals who met PTSD criteria for both $D S M-I V$ and $I C D-11$ ("DSM-IV and $I C D-11$ "), individuals who met $I C D$ 11 but not DSM-IV PTSD criteria ("ICD-11 only"), and individuals who met DSM-IV but not $I C D-11$ PTSD criteria (“DSM-IV only”). We planned chi-square tests to compare comorbidity estimates among non-overlapping groups (required for chi-square analyses) with sufficient sample size (>5 per cell; Yates, 1934). The two groups that met these criteria were the "All ICD11 " and "DSM-IV only" groups.

\section{Results}

Table 2 lists the estimated prevalence of PTSD across the three samples using the various diagnostic algorithms. Across all three samples, ICD-10 yielded the highest prevalence; ICD-11 produced the lowest, and the DSM-5 (Samples 1 and 2) and DSM-IV (Sample 3) estimates fell between those two. Table 3 shows the pattern of diagnostic concordance/discordance between ICD-11 and DSM-5 in Samples 1 and 2. Of 120 participants in Sample 1 who met PTSD criteria according to either $D S M-5, I C D-11$, or both, 75 (62.5\%) had discordant diagnoses, meaning that 
they met criteria for PTSD according to one diagnostic system but not the other. The McNemar's test indicated that the proportion of participants meeting criteria for ICD-11 in Sample 1 was significantly less than that for the $D S M-5$ definition, $\chi^{2}(1, \mathrm{~N}=2695)=14.52, p<$ 0.001, $\varphi=0.07$. In Sample 2, of 137 participants who met criteria according to either DSM-5, ICD-11, or both, $38(27.7 \%)$ had discordant diagnoses. Again, the McNemar's test indicated that the prevalence of $I C D-11$ PTSD was significantly less than that of $D S M-5 \operatorname{PTSD}, \chi^{2}(1, \mathrm{~N}=323)$ $=5.16, p=0.03, \varphi=0.13$. In both samples, the majority of discrepancies across the two definitions involved instances in which a participant met criteria for PTSD under the DSM-5 definition but did not meet criteria according to $I C D-11$. Specifically, in Sample 1, 99 participants met criteria for past-month DSM-5 PTSD but 54 of them (54.7\%) did not meet criteria using the $I C D-11$ definition. Similarly, in Sample 2, 125 participants met criteria for past-month DSM-5 PTSD but 26 (20.8\%) did not meet under the ICD-11 definition. Conversely, of the 66 individuals in Sample 1 who met criteria for past-month PTSD under ICD-11, 21 (31.8\%) did not meet under DSM-5 whereas, of the 111 participants in Sample 2 who met according $I C D-11$ criteria, $12(10.8 \%)$ did not meet under DSM-5.

We then sought to identify which component(s) of the two diagnostic algorithms contributed to lower prevalence estimates under $I C D-11$ relative to $D S M$-5. In Sample 1, there were 54 cases who met criteria for DSM-5 but not $I C D-11$. Of these, 32 (59.6\%) did not endorse at least one of the two requisite $I C D-11$ re-experiencing symptoms (nightmares or flashbacks), and $30(55.9 \%)$ did not endorse at least one of the requisite hyperarousal symptoms (hypervigilance or startle); of these, 8 (15.6\%) failed to meet both the ICD-11 re-experiencing and hyperarousal criteria. In Sample 2, we found that 15 (57.7\%) of those who met for DSM-5 did not meet criteria for $I C D-11$ because they did not endorse either nightmares or flashbacks, 12 
(46.2\%) did not meet for $I C D-11$ due to the absence of hypervigilance or startle, and $1(3.8 \%)$ did not meet based on lack of endorsement of both re-experiencing and hyperarousal symptoms.

Finally, we evaluated the hypothesis that eliminating the "non-specific symptoms" by paring the definition down to core symptoms would reduce comorbidity by examining patterns of comorbidity prevalence for ICD-11 compared with DSM-IV PTSD diagnoses. Table 4 presents the prevalence of comorbid alcohol abuse/dependence, generalized anxiety disorder, major depressive episode and panic disorder across the different PTSD definitions. Prevalence of comorbid conditions was very similar (within three percentage points) for individuals diagnosed with PTSD according to ICD-11 (“all ICD-11") compared with DSM-IV (“all DSM-IV”). We also ran chi-square tests comparing non-overlapping groups of individuals with PTSD according to $I C D-11$ ("all $I C D-11$ ") with individuals with PTSD according to DSM-IV but not $I C D-11$ ("DSM-IV only"). The only significant difference between the "all $I C D-11$ " and "DSM-IV only" groups occurred for alcohol abuse/dependence, which was significantly more common in the $I C D-11$ group $\left(12.2 \%\right.$ vs. $4.3 \%$ in $D S M-I V$ only), $\chi^{2}(1, \mathrm{~N}=273)=4.3, p=0.04, \varphi=0.13$. The prevalence of comorbid generalized anxiety disorder, major depressive episode, and panic disorder did not differ by group, $\chi^{2} \mathrm{~s}<0.5, n s$.

\section{Discussion}

This study examined the impact of changes proposed for the PTSD diagnosis in ICD-11 by comparing estimates of PTSD prevalence derived using ICD-11, ICD-10, DSM-IV, and DSM5 definitions of the disorder. We also tested the hypothesis that these changes would reduce the level of comorbidity associated with the diagnosis. Our analyses revealed that, across three samples, the estimated prevalence of PTSD varied considerably as a function of the diagnostic definition. ICD-10, by virtue of not requiring functional impairment, yielded the highest 
prevalence in each sample. This result is consistent with findings of prior studies that compared the ICD-10 and DSM-IV criteria for PTSD (Andrews et al., 1999; Peters et al., 1999; Rosenman, 2002) and underscores the importance of functional impairment in defining the diagnosis. In contrast, the $I C D-11$ diagnostic algorithm yielded prevalence estimates between 25 and 50 percent lower than ICD-10 and between 10 and 30 percent lower than the DSM-5 (Samples 1 \& 2) or DSM-IV (Sample 3) definitions. We also compared the concordance between the DSM-5 and $I C D-11$ diagnoses, and found that a significant proportion of individuals who would be diagnosed with PTSD according to one set of criteria would not be diagnosed with PTSD according to the other set of criteria (62.5\% in Study 1 and $27.7 \%$ in Study 2). The majority of these discrepancies were due to individuals who met PTSD criteria according to DSM-5 but not $I C D-11$, consistent with our findings of lower $I C D-11$ prevalence. In the future, $D S M-5$ and ICD-11 may be used concurrently in both clinical and research settings. The discordance raises doubts about the interchangeability of these two diagnoses and new questions about what distinguishes individuals who are diagnosed with PTSD according to one system but not the other.

Analyses that examined which component(s) of the two diagnostic algorithms contributed to the lower prevalence in $I C D-11$ revealed that the re-experiencing and hyperarousal clusters were equally likely to account for the discrepancies. These findings are consistent with those of O’Donnell et al. (2014) and suggest that by narrowing the definitions of re-experiencing and hyperarousal symptoms in $D S M-5$, the $I C D-11$ diagnosis may capture substantially fewer cases with clinically significant PTSD symptomatology (but see also Stein et al., 2014). The WHO workgroup aimed to restrict re-experiencing symptoms to those in which the traumatic event is "not only remembered, but experienced as occurring again" (Maercker, Brewin, Bryant, Cloitre, 
Reed, et al., 2013). Under $I C D-11$, physiological or emotional distress upon exposure to trauma-related reminders would be insufficient to meet the re-experiencing criterion. The eliminated symptoms are primary targets of exposure-based treatments (e.g., Foa et al., 2009) and conceptual cornerstones for fear-conditioning models and psychophysiological assessment methods in PTSD research (Keane et al., 1998; Pole, 2007). The more narrow definition and exclusion of these two symptoms represents a significant departure from current conceptualizations of traumatic re-experiencing. Additionally, from an assessment perspective, emphasizing symptoms in the domains of unconscious experience (nightmares) and dissociation (flashbacks) while excluding symptoms that are more readily reportable and observable (distress upon exposure) may introduce new problems of reliability.

The DSM-5 workgroup considered but rejected the circumscribed approach embodied in the ICD-11 proposal because it eliminated too many clinically significant components of the syndrome. To extend the $I C D-11$ argument to medical diseases, one would never include fever, pain, or edema as indicators of any diagnosis because they are found in so many different diseases (Friedman, 2013). Thus, the narrow versus broad approaches of ICD-11 and DSM-5, respectively, represent different conceptual models of PTSD and opposing beliefs about the clinical utility of such different diagnostic schemes. Although it is conceivable that the narrow approach would confer benefits such as increased discriminant validity or clinical utility, our findings indicate that the simplified set of criteria also would also come with a cost: failure to identify some individuals with clinically significant PTSD symptoms.

The second aim of this study was to test the $I C D-11$ workgroup's prediction that eliminating the "non-specific" symptoms of PTSD would reduce the level of comorbidity with other disorders, especially depression. Analysis of data from a carefully-assessed sample of over 
700 veterans and their partners showed no substantial reduction in comorbidity of alcohol abuse/dependence, generalized anxiety disorder, major depressive episode and panic disorder using the $I C D-11$ definition compared with the $D S M-I V$ definition. But more fundamentally, we disagree with the notion that the high level of comorbidity between PTSD and other disorders reflects a problem with the definition of the disorder that should be fixed by dropping symptoms. Eliminating overlapping symptoms has not emerged as an effective strategy for reducing comorbidity (Elhai et al., 2008; Grubaugh et al., 2010). Moreover, comorbidity is ubiquitous in mental illness and widely thought to be a reflection of the dimensional structure of psychopathology whereby broad classes of symptoms covary as a function of latent brainbehavior traits - attempting to eliminate it by redefining the construct and removing clinically relevant symptoms may prove counterproductive. Previous research suggests that one approach to addressing the challenges associated with diagnostic heterogeneity is to identify clinically and scientifically meaningful subtypes within samples of individuals with the diagnosis (Miller et al., 2004; Wolf et al. 2012).

That said, there are attractive aspects of the $I C D-11$ proposal. The working group has recommended a separate grouping of disorders specifically associated with stress rather than combining them with the anxiety disorders as has historically been the case in both diagnostic systems. As noted previously, we believe this to better reflect the causal role of trauma in the etiology of these disorders as well as the extensive phenotypic heterogeneity observed in samples with posttraumatic psychopathology (Miller et al., 2009; Resick and Miller, 2009). Furthermore, we agree with Maercker, Brewin, and others (Brewin, 2013; Maercker, Brewin, Bryant, Cloitre, Reed, et al., 2013; Maercker, Brewin, Bryant, Cloitre, van Ommeren, et al., 2013) that reexperiencing symptoms are the cardinal features of PTSD and that the avoidance symptoms are 
highly intertwined with them. Less convincing are arguments for defining hypervigilance and startle as pathognomonic to PTSD (Brewin, 2013; Maercker, Brewin, Bryant, Cloitre, Reed, et al., 2013; Maercker, Brewin, Bryant, Cloitre, van Ommeren, et al., 2013). Hypervigilance is a salient feature of panic disorder, simple phobia, and trait fearfulness so is, therefore, by no means unique to PTSD. Similarly, exaggerated startle has been observed in many other patient samples including those with panic disorder and social phobia (e.g., Grillon et al., 2008; Larsen et al., 2002; Melzig et al., 2007), obsessive-compulsive disorder (Kumari et al., 2001), and individuals with a familial risk for depression (Grillon et al., 2005). Further, while some research suggests that these symptoms may indeed be less saturated with "general distress variance" compared to other non-specific symptoms, to our knowledge, no study to date provides evidence for the more specific and improved associations between these symptoms and other putative indicators of threat and hyperarousal that would support the discriminant validity of this model over others (Miller, 2010; Miller et al., 2010).

Conclusions from this study should be considered in light of certain limitations. First, Samples 1 and 2 were based on internet surveys using a newly-developed instrument that has yet to undergo validation in direct relation to a clinical interview, the proposed $I C D-11$ criteria include a third re-experiencing symptom (vivid intrusive memories including fear or horror) which has not yet been examined empirically, and no comorbidity variables were available in the first two datasets. Second, the same survey was used to derive DSM-5 and ICD-11-defined PTSD (as opposed to independent DSM-5 and ICD-11 surveys) and this could have inflated concordance estimates. Third, Sample 3 featured diagnostic information derived from clinical interviews but those interviews were based on DSM-IV criteria, rather than DSM-5, and we did not have a large enough sample of individuals with PTSD according to $I C D-11$ but not DSM-IV 
to include this group in statistical comparisons. Fourth, Sample 1 was a national U.S.

community sample whereas Samples 2 and 3 were not national samples of veterans; the extent to which these findings will generalize to the entire veteran population or to other populations (i.e., outside the U.S.) is not certain. Finally, we did not address other aspects of the WHO Working Group's proposal, most notably, their plan for a complex PTSD diagnosis, though we have previously reported results calling into question the distinction between PTSD and complex PTSD (Wolf et al., 2015).

To conclude, the proposed $I C D-11$ criteria represent a major revision to the definition of PTSD and have stimulated new debate about the diagnosis. While one can debate the advantages and disadvantages of constraining the diagnosis to a narrower set of symptoms, the findings of this study demonstrate that doing so would substantially reduce the number of individuals reporting clinically significant symptoms who would meet criteria for the disorder. The public health and policy implications of a PTSD diagnosis that would yield substantially lower estimates of PTSD prevalence and caseness is concerning because of the potential impact on services available to those who are symptomatic. We suspect that this was not the intent of the ICD-11 workgroup and hope that these findings will stimulate investigation into the clinical, scientific, and policy implications of redefining the PTSD diagnosis. 


\section{Acknowledgment}

Data collection was funded by an American Psychiatric Association DSM grant to Dean Kilpatrick and the U.S. Department of Veterans Affairs. Manuscript preparation was supported by a VA Merit Review grant (5I01CX000431) awarded to Mark Miller, a VA Clinical Sciences Research and Development Program Career Development Award to Erika Wolf, funding from the National Institute of Mental Health (1R01MH095737-01A1), Department of Defense (W81XWH-12-2-0117-PTSD-IIRA-INT), Defense Advanced Research Programs Agency (N66001-11-C-4006) and Department of Veterans Affairs (Cooperative Studies Program \# 591) awarded to Dr. Marx, and funding from the Department of Defense (W81XWH-10-2-0181) awarded to Drs. Marx and Keane. The authors have no conflicts of interest to report. Drs. Friedman and Kilpatrick served on the Trauma/Stressor-Related and Dissociative Disorders SubWork Group of the Anxiety Disorder Work Group of DSM-5. Contents are solely the responsibility of the authors and views expressed do not represent those of the APA, the U.S. Department of Veterans Affairs, the U.S. Government, or other agencies supporting this research. 


\section{References}

American Psychiatric Association, 2013. Diagnostic and Statistical Manual of Mental Disorders, fifth ed. American Psychiatric Association, Arlington, VA.

Andrews, G., Slade, T., Peters, L., 1999. Classification in psychiatry: ICD-10 versus DSM-IV. Brit. J. Psychiatry 174, 3-5. doi:10.1192/bjp.174.1.3

Blake, D.D., Weathers, F.W., Nagy, L.M., Kaloupek, D.G., Gusman, F.D., Charney, D.S., Keane, T.M., 1995. The development of a clinician-administered PTSD scale. J. Trauma. Stress. 8(1), 75-90. doi:10.1002/jts.2490080106

Brewin, C.R., 2013. "I wouldn't start from here”: An alternative perspective on PTSD from ICD11. J. Trauma. Stress. 26(5), 557-559. doi:10.1002/jts.21843

Brewin, C.R, Lanius, R.A., Novac, A., Schnyder, U., Galea, S., 2009. Reformulating PTSD for DSM-V: Life after Criterion A. J. Trauma. Stress. 22(5), 366-373. doi:10.1002/jts.20443

Cloitre, M., Garvert, D.W., Brewin, C.R., Bryant, R.A., Maercker, A., 2013. Evidence for proposed ICD-11 PTSD and complex PTSD: A latent profile analysis. Eur. J. psychotraumatol. 4. doi: $10.3402 /$ ejpt.v4i0.20706

Elhai, J.D., Grubaugh, A.L., Kashdan, T.B., Frueh, B.C., 2008. Empirical examination of a proposed refinement to DSM-IV posttraumatic stress disorder symptom criteria using the National Comorbidity Survey Replication data. J. Clin. Psych. 69(4), 597-603. doi: $\underline{\text { 10.4088/JCP.v69n0411 }}$

First, M.B., Spitzer, R.L., Gibbon, M., Williams, J.B.W., 1994. Structured Clinical Interview for Axis I DSM-IV Disorders. Biometrics Research, New York.

Foa, E.B., Keane, T.M., Friedman, M.J., Cohen, J.A., 2009. Effective treatments for PTSD, second ed. Guilford Press, New York. 
Friedman, M.J., 2013. Finalizing PTSD in DSM-5: Getting here from there and where to go next. J. Trauma. Stress. 26(5), 548-556. doi:10.1002/jts.21840

Grillon, C., Lissek, S., Rabin, S., McDowell, D., Dvir, S., Pine, D. S., 2008. Increased anxiety during anticipation of unpredictable but not predictable aversive stimuli as a psychophysiologic marker of panic disorder. Am. J. Psychiatry 165(7), 898-904. doi:10.1176/appi.ajp.2007.07101581

Grillon, C., Warner, V., Hille, J., Merikangas, K.R., Bruder, G.E., Tenke, C.E., ...Weissman, M. M., 2005. Families at high and low risk for depression: A three-generation startle study. Biol. Psychiatry 57(9), 953-960. doi:10.1016/j.biopsych.2005.01.045

Grubaugh, A.L., Long, M.E., Elhai, J.D., Frueh, B.C., Magruder, K.M. 2010. An examination of the construct validity of posttraumatic stress disorder with veterans using a revised criterion set. Behav Res Ther, 48(9), 909-914. doi:10.1016/j.brat.2010.05.019

Keane, T.M., Kolb, L.C., Kaloupek, D.G., Orr, S.P., Blanchard, E.B., Thomas, R.G., ...Lavori, P.W., 1998. Utility of psychophysiological measurement in the diagnosis of posttraumatic stress disorder: Results from a Department of Veterans Affairs cooperative study. J. Consult. Clin. Psychol. 66(6), 914-923. doi:10.1037/0022-006X.66.6.914

Kilpatrick, D.G., Resnick, H.S., Baber, B., Guille, C., Gros, K., 2011. The National Stressful Events Web Survey (NSES-W). Medical University of South Carolina, Charleston, SC.

Kilpatrick, D., Resnick, H.S., Milanak, M.E., Miller, M.W., Keyes, K.M., Friedman, M.J., 2013. National estimates of exposure to traumatic events and PTSD prevalence using DSM-IV and DSM-5 Criteria. J. Trauma. Stress 26(5), 537-547. doi:10.1002/jts.21848

Kubany, E.S., Leisen, M.B., Kaplan, A.S., Watson, S.B., Haynes, S.N., Owens, J.A., Burns, K., 2000. Development and preliminary validation of a brief broad-spectrum measure of 
trauma exposure: The Traumatic Life Events Questionnaire. Psychol. Assess. 12(2), 210224. doi:10.1037/1040-3590.12.2.210

Kumari, V., Kaviani, H., Raven, P.W., Gray, J.A., Checkley, S.A., 2001. Enhanced startle reactions to acoustic stimuli in patients with obsessive-compulsive disorder. Am. J. Psychiatry 158(1), 134-136. doi:10.1176/appi.ajp.158.1.134

Larsen, D.K., Norton, G.R., Walker, J.R., Stein, M.B., 2002. Analysis of startle responses in patients with panic disorder and social phobia. Cogn. Behav. Therapy 31(4), 156-169. doi:10.1080/165060702321138555

Logue, M.W., Baldwin, C., Guffanti, G., Melista, E., Wolf, E.J., Reardon, A.F., ... Miller, M.W., 2013. A genome-wide association study of post-traumatic stress disorder identifies the retinoid-related orphan receptor alpha (RORA) gene as a significant risk locus. Molecular psychiatry 18(8), 937-942. doi: 10.1038/mp.2012.113

Maercker, A., Brewin, C.R., Bryant, R.A., Cloitre, M., Reed, G.M., van Ommeren, M., ...Saxena, S., 2013. Proposals for mental disorders specifically associated with stress in the International Classification of Diseases-11. Lancet 381(9878), 1683-1685. doi:10.1016/S0140-6736(12)62191-6

Maercker, A., Brewin, C.R., Bryant, R.A., Cloitre, M., van Ommeren, M., Jones, L.M., ...Reed, G.M., 2013. Diagnosis and classification of disorders specifically associated with stress: Proposals for ICD-11. World Psychiatry 12(3), 198-206. doi:10.1002/wps.20057

Melzig, C.A., Weike, A.I., Zimmermann, J., Hamm, A.O., 2007. Startle reflex modulation and autonomic responding during anxious apprehension in panic disorder patients. Psychophysiol. 44(6), 846-854. doi:10.1111/j.1469-8986.2007.00560.x 
Miller, M.W., 2010. On comparing competing models of PTSD: response to Simms. J. Trauma. Stress 23(5), 642-644. doi:10.1002/jts.20561

Miller, M.W., Kaloupek, D.G., Dillon, A.L., \& Keane, T.M. (2004). Externalizing and internalizing subtypes of combat-related PTSD: A replication and extension using the PSY-5 Scales. J. Abnorm. Psychol 113, 636-645. doi:10.1037/0021-843X.113.4.636

Miller, M.W., Resick, P.A., Keane, T.M., 2009. DSM-V: Should PTSD be in a class of its own? . British J. Psychiatry 194(1), 90. doi:10.1192/bjp.194.1.90

Miller, M.W., Wolf, E.J., Harrington, K., Brown, T.A., Kaloupek, D.G., Keane, T.M., 2010. An evaluation of competing models for the structure of PTSD symptoms using external measures of comorbidity. J. Trauma. Stress 23(5), 631-638. doi:10.1002/jts.20559

Miller, M.W., Wolf, E.J., Kilpatrick, D., Resnick, H., Marx, B.P., Holowka, D.W., ...Friedman, M.J., 2013. The prevalence and latent structure of proposed DSM-5 posttraumatic stress disorder symptoms in US national and veteran samples. Psychol. Trauma: Theory, Research, Practice, and Policy 5(6), 501-512. doi:10.1037/a0029730

Miller, M.W., Wolf, E.J., Logue, M., Baldwin, C., 2013. The retinoid-related orphan receptor alpha (RORA) gene and fear-related psychopathology. J. Affective Disorders 151(2), 702-708. doi:10.1016/j.jad.2013.07.022

Morina, N., Emmerik, A.A., Andrews, B., Brewin, C.R. (2014). Comparison of DSM-IV and proposed ICD-11 formulations of PTSD among civilian survivors of war and war veterans. J Trauma Stress 27(6), 647-654. doi:10.1002/jts.21969

O’Donnell, M.L., Alkemade, N., Nickerson, A., Creamer, M., McFarlane, A.C., Silove, D., ...Forbes, D., 2014. Impact of the diagnostic changes to post-traumatic stress disorder for 
PTSD-5 and the proposed changes to ICD-11. Brit. J. Psychiatry 205(3), 230-235. doi:10.1192/bjp.bp.113.135285

Peters, L., Slade, T., Andrews, G.A., 1999. A comparison of ICD-10 and DSM-IV criteria for posttraumatic stress disorder. J. Trauma. Stress 12(2), 335-343. doi:10.1023/A:1024732727414

Pole, N., 2007. The psychophysiology of posttraumatic stress disorder: A meta-analysis. Psychol. Bull. 133(5), 725-746. doi:10.1037/0033-2909.133.5.725

Reed, G.M., 2010. Toward ICD-11: Improving the clinical utility of WHO's International Classification of Mental Disorders. Prof. Psychol.: Research and Practice 41(6), 457-464. doi:10.1037/a0021701

Resick, P.A., Miller, M.W., 2009. Posttraumatic Stress Disorder: Anxiety or Traumatic-Stress Disorder? J. Trauma. Stress 22(5), 384-390. doi:10.1002/jts.20437

Rosen, G.M., Lilienfeld, S.O., Frueh, B.C., McHugh, P.R., Spitzer, R.L., 2010. Reflections of PTSD's future in DSM-V. British J. Psychiatry 197(5), 343-344. doi:10.1192/bjp.bp.110.079699

Rosen, R.C., Marx, B.P., Maserejian, N.N., Holowka, D.W., Gates, M.A., Sleeper, L.A., ...Keane, T.M., 2012. Project VALOR: Design and methods of a longitudinal registry of post-traumatic stress disorder (PTSD) in combat-exposed veterans in the Afghanistan and Iraqi military theaters of operations. Int. J. Meth. Psychiatric Research 21(1), 5-16. doi:10.1002/mpr.355

Rosenman, S., 2002. Trauma and posttraumatic stress disorder in Australia: Findings in the population sample of the Australian National Survey of Mental Health and Wellbeing. 
Australian and New Zealand J. Psychiatry 36(4), 515-520. doi:10.1046/j.14401614.2002.01039.x

Stammel, N., Abbing, E.M., Heeke, C., Knaevelsrud, C., 2015. Applicability of the ICD-11 proposal for PTSD: A comparison of prevalence and comorbidity rates with the DSM-IV PTSD classification in two post-conflict samples. Eur. J. psychotraumatol. 6. doi: 10.3402/ejpt.v6.27070

Stein, D.J., McLaughlin, K.A., Koenen, K.C., Atwoli, L., Friedman, M.J., Hill, E.D., ...Kessler, R.C., 2014. DSM-5 and ICD-11 definitions of posttraumatic stress disorder: Investigating “narrow" and "broad” approaches. Depres Anxiety. 31(6), 494-505. doi:10.1002/da.22279

Weathers, F.W., Litz, B.T., Herman, D.S., Huska, J.A., Keane, T.M., 1993. The PTSD Checklist (PCL): Reliability, validity, and diagnostic utility. Poster presented at Ninth Annual Conference of the ISTSS, San Antonio, TX.

Wolf, E.J., Miller, M.W., Kilpatrick, D., Resnick, H., Badour, C.L., Marx, B.P., ...Friedman, M., 2015. ICD-11 Complex PTSD in U.S. national and veteran samples: Prevalence and structural associations with PTSD. Clin. Psychol. Sci. 3, 215-229. doi: $10.1177 / 2167702614545480$

Wolf, E.J., Miller, M.W., Reardon, A.F., Ryabchenko, K., Castillo, D., Freund, R., 2012. A latent class analysis of dissociation and PTSD: Evidence for a dissociative subtype. Arch. Gen. Psychiat. 69, 698-705. doi:10.1001/archgenpsychiatry.2011.1574.

World Health Organization., 1992. The ICD-10 Classification of Mental and Behavioural Disorders. World Health Organization, Geneva, Switzerland. 
Yates, F., 1934. Contingency tables involving small numbers and the $\chi^{2}$ test. J. R. Stat. Soc. 1(2), 217-235. doi: 10.2307/2983604 
Table 1.

Sample Characteristics across the Three Studies.

\begin{tabular}{|c|c|c|c|}
\hline & Study 1 & Study 2 & Study 3 \\
\hline$n$ & 2953 & 323 & 748 \\
\hline \multirow[t]{3}{*}{ Sample } & Population-based & Veterans & Veterans and \\
\hline & Community & & Partners \\
\hline & Sample & & \\
\hline Sex (\% Female) & $52 \%$ & $39 \%$ & $41 \%$ \\
\hline \multicolumn{4}{|l|}{ Age Distribution, $n, \underline{(\%)}$} \\
\hline $18-24$ & $332(11.3 \%)$ & $4(1.2 \%)$ & $17(2.3 \%)$ \\
\hline $25-34$ & $563(19.1 \%)$ & $101(31.3 \%)$ & $56(7.5 \%)$ \\
\hline $35-44$ & $508(17.2 \%)$ & $72(22.3 \%)$ & $106(14.2 \%)$ \\
\hline $45-54$ & $571(19.3 \%)$ & $72(22.3 \%)$ & $213(28.5 \%)$ \\
\hline $55-64$ & $488(16.5 \%)$ & $53(16.4 \%)$ & $327(43.7 \%)$ \\
\hline 65 or older & $490(16.6 \%)$ & $16(5.0 \%)$ & $29(3.9 \%)$ \\
\hline \multicolumn{4}{|l|}{ Race } \\
\hline Caucasian/White & $75 \%$ & $80 \%$ & $81 \%$ \\
\hline African American/Black & $12 \%$ & $16 \%$ & $12 \%$ \\
\hline
\end{tabular}


American Indian/Alaskan Native

Asian/Pacific Islander

Multiracial

"Other" or "Unknown"

Ethnicity (\% Hispanic)

Exposure to one or more DSM-5

Criterion A events

$$
2 \%
$$

$4 \%$

$9 \%$

$$
5 \%
$$

$1 \%$

$2 \%$

$$
5 \%
$$

$17 \%$

$5 \%$

$15.5 \%$

$89.7 \%$

$100 \%$

$100 \%$

Note. Totals for race may sum to greater than $100 \%$ because participants could select more than one racial ancestry category. Five participants in Study 2 did not report age. 
Table 2

Prevalence Estimates (\%) for DSM and ICD Past-Month PTSD across the 3 samples

DSM-IV DSM-5 ICD-10 ICD-11

Sample 1 (community; $\mathrm{N}=2,695$ )

3.8

3.7

4.6

2.4

Sample 2 (veterans; $\mathrm{N}=323$ )

38.7

38.7

45.5

34.4

Sample 3 (veterans and partners;

35.3

$-\quad 38.0$

25.3

$$
\mathrm{N}=748)
$$

Note. DSM = Diagnostic and Statistical Manual; ICD = International Classification of Diseases; PTSD = posttraumatic stress disorder. Using DSM-IV Criterion A exposure resulted in 67 ICD11 PTSD cases as opposed to 66 ICD-11 cases when requiring exposure to a DSM-5 Criterion A event. 
Table 3

Concordance between Past-Month ICD-11 and Past-Month DSM-5 PTSD Diagnoses for Samples 1 (U.S. National) \& 2 (VA PTSD Clinic Sample)

DSM-5 Diagnosis

Negative $\quad$ Positive $\quad$ Total

Sample 1

(Community)

\begin{tabular}{|rrrr} 
Negative & $2,575(95.6)$ & $54(2.0)$ & $2,629(97.6)$ \\
Positive & $\ldots 21(0.8)$ & $45(1.7)$ & $\quad 66(2.4)$ \\
Total & $2,596(96.3)$ & $99(3.7)$ & $2,695(100.0)$
\end{tabular}

Sample 2

ICD-11

(VA)

$\begin{array}{cccc}\text { Negative } & 182(57.0) & 26(8.1) & 208(65.2) \\ \text { Positive } & \quad 12(3.7) & -99(31.0) & 111(34.8) \\ \text { Total } & 194(60.8) & 125(39.2) & 319(100)\end{array}$

Note. DSM = Diagnostic and Statistical Manual; ICD = International Classification of Diseases; PTSD = posttraumatic stress disorder; Values in each cell are numbers of participants followed be the percentage of total in parentheses. Diagnoses were based on symptoms endorsed moderately severe or higher (i.e., 3 or greater on a 5 point severity scale). In Sample 2, 4 subjects 
had missing data that precluded calculation of these cross-tabs so percentages differ slightly from Table 2. 
Table 4

Sample 3 Current Psychiatric Comorbidity Prevalence (\%) for Cases Meeting Criteria for the DSM-IV versus ICD-11 PTSD Diagnosis

\begin{tabular}{rccccc}
\hline Diagnosis & All & All & DSM-IV & ICD-11 & DSM-IV \\
& DSM-IV & ICD-11 & only & only & and ICD-11 \\
\hline Alcohol & $25 / 253$ & $22 / 181$ & $4 / 92$ & $1 / 20$ & $21 / 161$ \\
Abuse/Dependence & $(9.9 \%)$ & $(12.2 \%)$ & $(4.3 \%)$ & $(5.0 \%)$ & $(13.0 \%)$ \\
Generalized & $34 / 253$ & $24 / 181$ & $12 / 92$ & $2 / 20$ & $22 / 161$ \\
Anxiety Disorder & $(13.4 \%)$ & $(13.3 \%)$ & $(13.0 \%)$ & $(10.0 \%)$ & $(13.7 \%)$ \\
Major Depressive & $93 / 254$ & $65 / 183$ & $29 / 92$ & $1 / 21$ & $64 / 162$ \\
Episode & $(36.6 \%)$ & $(35.5 \%)$ & $(31.5 \%)$ & $(4.8 \%)$ & $(39.5 \%)$ \\
Panic Disorder & $9 / 252$ & $6 / 180$ & $4 / 92$ & $1 / 20$ & $5 / 160$ \\
& $(3.6 \%)$ & $(3.3 \%)$ & $(4.3 \%)$ & $(5.0 \%)$ & $(3.1 \%)$ \\
\hline
\end{tabular}

Note. "All DSM-IV" and "All ICD-11" refer to all individuals with PTSD according to the respective diagnostic system. "DSM-IV only" and "ICD-11 only" refers to individuals with discordant diagnoses (PTSD according to one diagnostic system but not the other). "DSM-IV and ICD-11" refers to the group of individuals with PTSD according to both systems.

Numerator values represent the number of participants diagnosed with the given comorbidity, whereas denominators represent the number of participants diagnosed with PTSD according to the respective diagnostic system. Denominators differ by cell because comorbidity data were missing for some participants; missing data represented $<5 \%$ of the data collected in any given cell. 